\title{
On Divisors of Odd Perfect Numbers
}

\author{
By Joseph B. Muskat
}

A perfect number is a positive integer the sum of whose divisors is equal to twice the number itself. Twenty-three even perfect numbers have been discovered to date [2]. No odd perfect number has yet been found, but various restrictions which an odd perfect number must satisfy have been established. For a summary, see [7].

For a perfect number $n, \sigma(n)=2 n$, where $\sigma(n)$ denotes the sum of the divisors of $n$. Let

$$
n=\prod f_{j}^{e_{j}}
$$

where the $f_{j}$ are distinct primes. Since $\sigma$ is a multiplicative function $[8, \mathrm{p} .88]$,

$$
2 n=2 \prod f_{j}^{e_{j}}=\sigma(n)=\prod \sigma\left(f_{j}^{e_{j}}\right) .
$$

Any divisor of the right side of (1) must divide $2 n$

is an immediate consequence of (1). For example if 9 , but not 27 , divides $n$, then $\sigma\left(3^{2}\right)=13$ divides $n$.

Euler deduced from (1) that $n$ must be of the form

$$
n=p^{a} \prod_{i=1}^{r} q_{i}^{2 b_{i}}, \quad \text { where } \quad p \equiv a \equiv 1 \quad(\bmod 4)
$$

and $p$ and the $q_{i}$ denote distinct primes [1, pp. 14-15]. Kühnel [5] and others have proved that $r \geqq 5$.

Using these and other results, Kanold showed that there are no odd perfect numbers less than $10^{20}[3]$. This superseded a bound of $10^{18}$, obtained by the author $[8$, p. $359 \mathrm{~b}]$ with the help of the following:

\section{(4) Any odd perfect number must be divisible by a prime power greater than $10^{8}$.}

Ore studied numbers whose harmonic means are integers, and showed that perfect numbers have this property [9]. W. H. Mills demonstrated that any odd number with an integral harmonic mean must have a prime power factor greater than $10^{7}$. This bound in Mills' (unpublished) calculation arose from the limited range of D. N. Lehmer's factor table [6] which Mills utilized. The author (as a part of his undergraduate thesis which was supervised by Professor Ore) extended Mills' result in the special case of odd perfect numbers with the aid of tables of Kraitchik [4, pp. 89, 91, 152-159] to obtain (4).

More recently, the help of digital computers was enlisted to prove the following:

Theorem. Any odd perfect number must be divisible by a prime power greater than $10^{12}$.

Outline of Proof. Assume that every prime power factor of $n$ is less than $10^{12}$. Steuerwald showed that at least one of the $b_{i}$ in equation (3) must be greater than 1 [10]. The corresponding $q_{i}$, therefore, must be less than 1000 .

It was found that for each $f^{e}$, where $f$ is a prime $<1000$ and $f^{e}<10^{12}$, eventually at least one of the following three contradictions develop by (repeated, if necessary) reference to (2):

Received July 19, 1965. 
TABLE 1

\begin{tabular}{|c|c|c|c|c|c|c|c|c|c|c|c|c|}
\hline Phase & \multicolumn{12}{|c|}{ Primes Eliminated } \\
\hline 1 & 127 & 271 & & & & & & & & & & \\
\hline 2 & 911 & & & & & & & & & & & \\
\hline 3 & 19 & & & & & & & & & & & \\
\hline 4 & 239 & 311 & 443 & 691 & 839 & 859 & & & & & & \\
\hline 5 & 179 & 919 & & & & & & & & & & \\
\hline $\begin{array}{l}6 \\
7\end{array}$ & $\begin{array}{r}11 \\
163\end{array}$ & 467 & 619 & & 883 & 971 & & & & & & \\
\hline 8 & 71 & 547 & & 001 & & & & & & & & \\
\hline 9 & 587 & 593 & 709 & 1093 & & & & & & & & \\
\hline 10 & 151 & 227 & & & & & & & & & & \\
\hline 11 & 571 & & & & & & & & & & & \\
\hline 12 & 109 & 461 & & & & & & & & & & \\
\hline 13 & 263 & 499 & 653 & & & & & & & & & \\
\hline 14 & 7 & 359 & & & & & & & & & & \\
\hline \multirow[t]{2}{*}{15} & 37 & 97 & 191 & 331 & 347 & 431 & 487 & 599 & 683 & 739 & 751 & 787 \\
\hline & 823 & 863 & 907 & 977 & & & & & & & & \\
\hline 16 & 31 & 47 & 193 & 379 & 433 & 491 & 569 & 643 & 719 & 997 & & \\
\hline 17 & 61 & 281 & 293 & 349 & 557 & 631 & & & & & & \\
\hline 18 & 13 & 59 & 131 & & & & & & & & & \\
\hline 19 & 23 & 113 & 167 & 233 & 337 & 353 & 367 & 389 & 419 & 503 & 523 & 607 \\
\hline 20 & $\begin{array}{r}009 \\
3\end{array}$ & 137 & $\begin{array}{l}881 \\
229\end{array}$ & 283 & 373 & 677 & 733 & & & & & \\
\hline \multirow[t]{3}{*}{21} & 5 & 29 & 43 & 53 & 73 & 79 & 89 & 101 & 103 & 149 & 173 & 181 \\
\hline & 199 & 223 & 241 & 257 & 269 & 307 & 317 & 383 & 401 & 439 & 449 & 457 \\
\hline & 521 & 617 & 641 & 727 & 773 & 809 & 821 & 827 & 853 & 937 & 967 & \\
\hline \multirow[t]{3}{*}{22} & 17 & 41 & 83 & 157 & 211 & 251 & 397 & 409 & 479 & 509 & 541 & 601 \\
\hline & 613 & 661 & 701 & 743 & 761 & 811 & 829 & 877 & 881 & 941 & 947 & 983 \\
\hline & 991 & & & & & & & & & & & \\
\hline 23 & 67 & 107 & 139 & 197 & 313 & 421 & 463 & 577 & 647 & 769 & 797 & 929 \\
\hline 24 & $\begin{array}{l}953 \\
277\end{array}$ & 563 & 673 & & & & & & & & & \\
\hline
\end{tabular}

(a) The integer $n$ has a prime factor $F \equiv 3(\bmod 4), F>10^{6} . F$ has an even exponent by (3). But then $F^{2}>10^{12}$.

(b) A sequence of prime divisors develops that includes primes $G, H \equiv 1$ $(\bmod 4)$, where $G$ is assigned an odd exponent and $H>10^{6}$. By (3), $H$ must have an even exponent, and $H^{2}>10^{12}$.

(c) A prime factor $<1000$ (or 1093, which is specially included for convenience) that has been eliminated previously is encountered.

The proof was divided into twenty-four phases. A prime factor $f<1000$ (or 1093 ) is eliminated during phase $P+1$ if the previously eliminated primes upon which its exclusion depends include at least one prime in phase $P$. In order to shorten the proof, exclusions which depended upon previously eliminated primes were sought.

The 168 possible primes are eliminated successively in the order indicated in Table 1.

For reasons of space, only the first two phases of the proof are included here as Table 2. (The author will supply a copy of the complete proof upon request.) A copy has been placed in the UMT file of this journal. 
127

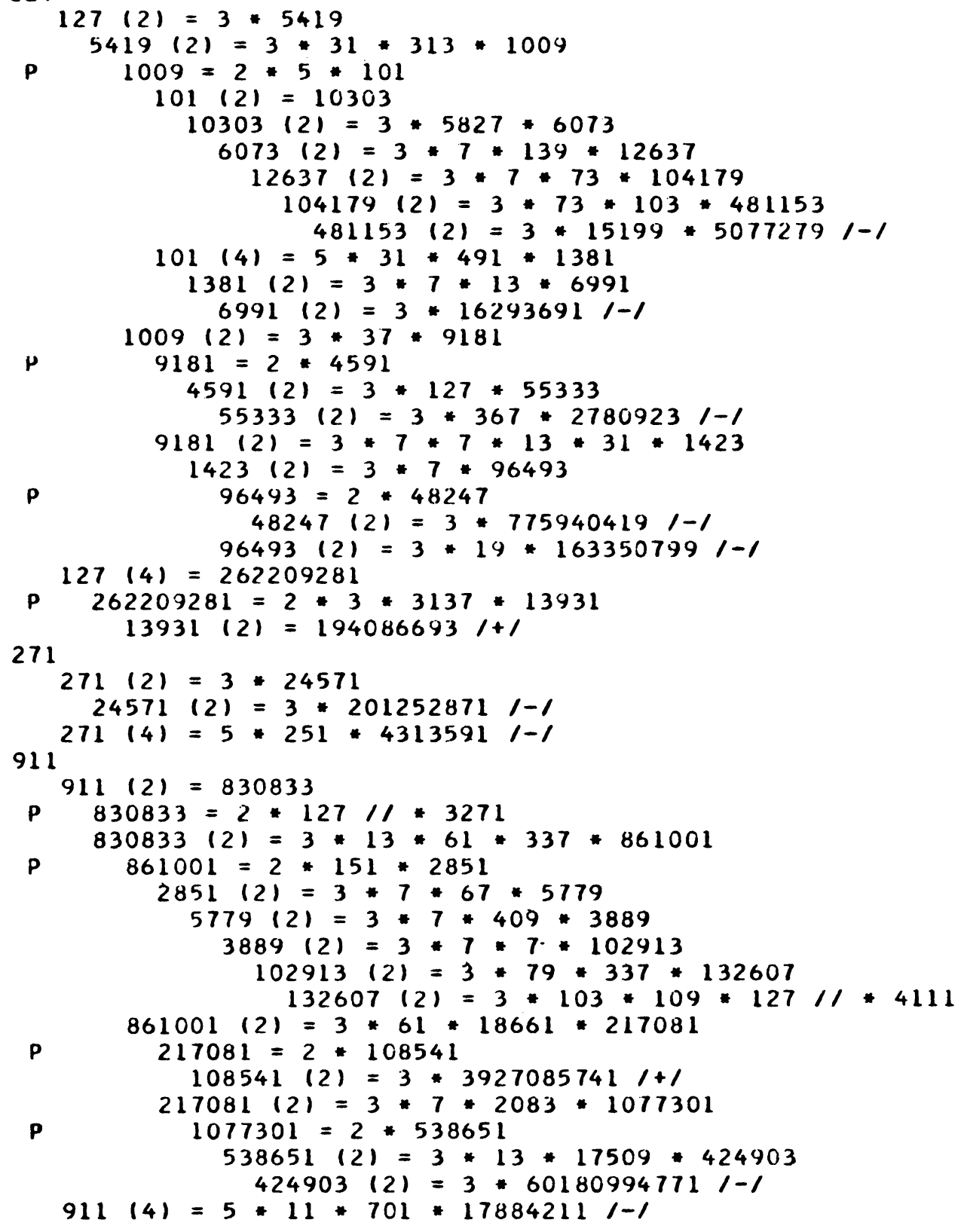

The proof was recorded on punched cards, so only a restricted set of characters was available. The second and third lines of the proof would appear in conventional notation as follows:

$$
\begin{aligned}
\sigma\left(127^{2}\right) & =3 \cdot 5419 \\
\sigma\left(5419^{2}\right) & =3 \cdot 31 \cdot 313 \cdot 1009
\end{aligned}
$$


The three criteria for exclusion, (a), (b), and (c), are marked by placing the symbols $/-/, /+/$, and $/ /$, respectively, after the prime.

For primes $\equiv 1(\bmod 4)$, the only odd exponent which had to be considered was 1 , as $\sigma(p)$ divides $\sigma\left(p^{2 m+1}\right)$. The prime with the odd exponent is preceded by the letter $P$.

With this result, Kanold's lower bound of $10^{20}$ for an odd perfect number can be raised. To produce a specific number as a bound, however, it is necessary to assemble various other restrictions upon odd perfect numbers. This is not being undertaken here, as M. Garcia has obtained (but not published) a yet higher bound.

The University of Pittsburgh's IBM 7070 and IBM 7090 digital computers were used to obtain prime factorizations and to check the accuracy and completeness of the proof. The author wishes to express his appreciation to the University of Pittsburgh's Computation and Data Processing Center for granting access to these computers. This facility is supported in part under National Science Foundation Grants G11309 and GP2310.

University of Pittsburgh

Pittsburgh, Pennsylvania

1. L. Euler, Opera Postuma, Vol. 1.

2. D. B. GILliEs, "Three new Mersenne primes and a statistical theory," Math. Comp., v. 18,1964 , pp. $93-95$. MR 28 *2990.

3. H.-J. KANOLD, “Über mehrfach vollkommene Zahlen II," J. Reine. Angew. Math., v. 197,1957, pp. 82-96. MR 18, 873. 1929.

4. M. KRA Iтchiк, Recherches Sur la Théorie des Nombres, Vol. II, Gauthier-Villars, Paris,

5. U. KüHNEL, "Verschärfung der notwendigen Bedingungen für die Existenz von ungeraden vollkommenen Zahlen," Math. $Z$., v. 52, 1949, pp. 202-211. MR 11, 714.

6. D. N. Lenmer, Factor Table for the First Ten Millions Containing the Smallest Factor of Every Number Not Divisible by 2, 3, 5, or 7 Between the Limits 0 and 10, 017,000, Carnegie Institute Publication 105, Washington, D. C., 1909.

7. P. J. MCCARTHY, "Odd perfect numbers," Scripta Math., v. 23, 1957, pp. 43-47. MR $21 * 21$. 1956.

8. O. Ore, Number Theory and its History (with supplement), McGraw-Hill, New York,

9. O. ORE, "On the averages of the divisors of a number," Amer. Math. Monthly, v. 55, 1948 , pp. 615-619. MR 10, 284.

10. R. STEUERWALD, "Verschärfung einer notwendigen Bedingung für die Existenz einer ungeraden vollkommenen Zahl," Sitzungsberichte der mathematisch-naturwissenschaftlichen Abteilung der Bayerischen Akademie der Wissenschaften zu München, Bayerischen Akademie der Wissenschaften, Munich, 1937, pp. 69-72.

\section{Solutions of the Diophantine Equations}

$$
x^{2}+y^{2}=l^{2}, y^{2}+z^{2}=m^{2}, z^{2}+x^{2}=n^{2}
$$

\section{By M. Lal and W. J. Blundon}

Introduction. The solution of the system of three equations of the second degree in six unknowns i.e. $x^{2}+y^{2}=l^{2}, y^{2}+z^{2}=m^{2}$ and $z^{2}+x^{2}=n^{2}$ is a classical Diophantine problem [1, p. 112]. The geometrical significance of this problem is to find a rectangular parallelepiped whose edges and face diagonals are all rational integers. If $x, y$ and $z$ are relatively prime in pairs the above system has no solution; otherwise there are infinitely many solutions.

Received May 25, 1965. 